Research Paper

\title{
Assessing the growth and recovery of Salmonella Enteritidis SE86 after sodium dichloroisocyanurate exposure
}

\author{
Fernanda Stoduto Ferreira, Mariana Bandeira Horvath, Eduardo Cesar Tondo \\ Laboratório de Microbiologia e Controle de Alimentos, Instituto de Ciência e Tecnologia de Alimentos, \\ Universidade Federal do Rio Grande do Sul, Porto Alegre, RS, Brazil.
}

Submitted: May 2, 2012; Approved: November 13, 2012.

\begin{abstract}
The objective of the present study was to assess the growth and the recovery of Salmonella $(S$.) Enteritidis SE86 in different diluents, culture media and using different plating methods after the exposure to $200 \mathrm{mg} / \mathrm{kg}$ sodium dichloroisocyanurate (NaDCC). Before and after NaDCC exposure, SE86 was cultured at $30^{\circ} \mathrm{C}$ and $7{ }^{\circ} \mathrm{C}$ in the following diluents: Peptone water (P), Saline solution $(\mathrm{SaS})$, Peptone water+Saline solution $(\mathrm{P}+\mathrm{SaS})$, Peptone water+Tween $80+$ Lecithin+Sodium thiosulfate $(\mathrm{P}+\mathrm{N})$ and Saline solution + Tween $80+$ Lecithin+Sodium thiosulfate $(\mathrm{SaS}+\mathrm{N})$. The SaS diluent was chosen because it was able to maintain cells viable without growth and was further used for plating SE86 on non selective medium (Tryptic Soy Agar-TSA) and on selective media (Mannitol Lysine Crystal Violet Brilliant Green Agar-MLCB; Brilliant Green Agar-BGA; Salmonella Shigella Agar-SS and Xylose Lysine Dextrose-XLD). The Thin Agar Layer method (TAL) i.e., selective media overlayed with non selective TSA was also evaluated. Results indicated that SE86 not exposed to $\mathrm{NaDCC}$ was able to grow in $\mathrm{P}, \mathrm{P}+\mathrm{N}, \mathrm{SaS}+\mathrm{N}$ and $\mathrm{P}+\mathrm{SaS}$, but not in $\mathrm{SaS}$, that was able to maintain cells viable. SE86 exposed to NaDCC demonstrated similar counts after dilution in SaS and the plating on non selective TSA, selective media MLCB, BGA, SS and XLD and on TAL media. SE86, $S$. Typhimurium and $S$. Bredeney, exposed or not exposed to NaDCC, showed no significant differences in counts on TSA, XLD and XLD overlayed with TSA, suggesting that all those media may be used to quantify NaDCC-exposed Salmonella by plating method.
\end{abstract}

Key words: Salmonella Enteritidis SE86, diluents, plating methods, stressed cells, sodium dichloroisocyanurate.

\section{Introduction}

Salmonellosis is one of the most important public health problems worldwide (Greig and Ravel et al., 2009; Tondo and Ritter, 2012). In Brazil, Salmonella spp. was identified as the main etiological agent causing foodborne diseases in the period of 1999 to 2011, being responsible for 1660 foodborne outbreaks investigated by the Brazilian Inspection Services (Brasil, 2011). Using pheno and genotyping methods (Oliveria et al., 2012; Geimba et al., 2004), a specific strain of $S$. Enteritidis (named SE86) was identified in more than $95 \%$ of the salmonellosis notified in State of Rio Grande do Sul (RS), southernmost State of Brazil, in the last years (Oliveira et al., 2012), arising an expressive interest in the investigation of this pathogen. Further studies demonstrated that SE86 presents high capability for thermal and acid adaptation after its exposure to sublethal $\mathrm{pH}$ and this capability was not verified in other Salmonella serovars (Malheiros et al., 2009). SE86 was also more resistant than other Salmonella serovars to 200, 400 and $800 \mathrm{mg} / \mathrm{kg}$ sodium hypochlorite and was able of forming biofilms on stainless steel and polyethylene (Tondo et al., 2010).

In the State of RS, between 2000 and 2002, insufficient cleanliness of utensils and equipments and cross contamination, together, were responsible for $14.15 \%$ of the foodborne outbreaks occurred in food services, indicating a

Send correspondence to E.C. Tondo. Laboratório de Microbiologia e Controle de Alimentos, Instituto de Ciência e Tecnologia de Alimentos ICTA/UFRGS, Universidade Federal do Rio Grande do Sul. Av. Bento Gonçalves 9500, Prédio 43212, Campus do Vale, Agronomia, Caixa Postal 15090, 91501-970 Porto Alegre, RS, Brasil. E-mail: tondo@ufrgs.br. 
possible deficiency in the hygiene of surfaces that come into contact with foods (Cunha et al., 2008). Microbiological analysis of utensil and equipment surfaces is an important tool for verifying the effectiveness of cleaning and disinfection procedures (Moore and Griffith, 2007), and the swab technique is the most suitable method for that (APHA, 2001). After disinfection of surfaces, many microorganisms are inactivated, but a part of the bacterial population may survive and remain in the VNC (Viable but Not Cultivable) condition. These bacteria may have been affected by the action of disinfectants, losing the ability to form colonies on selective media, however, they still be able to reproduce in non-selective media, being called "stressed" or "injured" (Wu and Fung, 2006; Wu, 2008). Stressed bacterial cells have great relevance to the food industry and food services, since they can not be detected in the microbiological analysis, but may be able to cause outbreaks or food quality problems, after their recovery. In this sense, the methods for analysis should allow recovery of bacterial cells, in order to obtain the number of microorganisms that are actually present in sample or on a surface (Jasson et al., 2007).

In food services and food industries, the chlorine compounds are among the most commonly disinfectants used for disinfection of surfaces (Peng et al., 2002; Rossoni and Gaylarde, 2000). In Brazil, probably the most used chlorine compounds are $200 \mathrm{mg} / \mathrm{kg}$ sodium hypochlorite or sodium dichloroisocyanurate, which can injure microorganisms present on food contact surfaces, avoiding their detection. Therefore, evaluating the effect of these sanitizers on important food pathogens such as $S$. Enteritidis SE86 is of great importance.

The aim of this study was to assess the growth and the recovery of S. Enteritidis SE86 in different diluents, media and by plating methods, after sodium dichloroisocyanurate exposure, aiming to identify adequate procedures to be used in swab sampling methods.

\section{Materials and Methods}

\section{Bacterial strains}

Strains of Salmonella enterica of three different serovars were used in this study. $S$. Enteritidis SE86 was isolated from a cabbage involved in a salmonellosis outbreak occurred in the State of RS, in 1999. This microorganism presents the same genotypic pattern of $S$. Enteritidis involved in more than $95 \%$ of the salmonellosis outbreaks occurred in the State of RS during the period of 1999 to 2006 (Oliveira et al., 2012). S. Typhimurium and $S$. Bredeney strains were isolated from pig fecal samples also in the State of RS. These strains were provided by Prof. Dr. Marisa Ribeiro de Itapema Cardoso of the Preventive Veterinary Department of Universidade Federal do Rio Grande do Sul (UFRGS) and there is no reports indicating the involvement of them with foodborne diseases in the State of
RS. Before experiments, the strains were stored at $-18^{\circ} \mathrm{C}$ in $30 \%(\mathrm{v} / \mathrm{v})$ glycerol, and working cultures were kept at $4{ }^{\circ} \mathrm{C}$ on Brain Heart Infusion Agar (BHI; Oxoid). Before use in the experiments, strains were activated in BHI broth at $37{ }^{\circ} \mathrm{C}$ for $18-24 \mathrm{~h}$, reaching a concentration of approximately $10^{8}$ cfu. $\mathrm{mL}^{-1}$. The initial concentration of cells was measure by plating the inoculum on BHI agar.

\section{Evaluation of growth of S. Enteritidis SE86 in different diluents}

Cultures of $S$. Enteritidis SE86 were activated in $9 \mathrm{~mL}$ of BHI at $37^{\circ} \mathrm{C}$ for $18-24 \mathrm{~h}$, reaching a concentration of approximately $10^{9}$ cfu. $\mathrm{mL}^{-1}$. Two experiments were conducted in parallel: one with $S$. Enteritidis SE86 not exposed to the disinfectant (Control) and other with $S$. Enteritidis SE86 exposed to $200 \mathrm{mg} / \mathrm{kg}$ NaDCC (Trade mark Kalyclean S313, Kalykim, Brazil). The exposure to NaDCC was performed by inoculating $1 \mathrm{~mL}$ of bacterial culture in $9 \mathrm{~mL}$ of $200 \mathrm{mg} \mathrm{kg}^{-1} \mathrm{NaDCC}$, for five minutes. This exposure time was used because it was able to reduce but not inactivate all the inoculated Salmonella. The exposure time and bacterial concentrations were assessed previously to the experiments of multiplication. The exposed and not exposed bacterial cultures were diluted until a concentration of $10^{8} \mathrm{cfu} \mathrm{mL}^{-1}$ in five different diluent solutions: $0.1 \%$ Peptone water $(\mathrm{P}) ; 0.85 \%$ Saline solution $(\mathrm{SaS}) ; 0.85 \%$ Saline solution $+0.1 \%$ Peptone water $(\mathrm{P}+\mathrm{SaS}) ; 0.1 \%$ Peptone water $+0.5 \%$ Tween $80+0.07 \%$ Lecithin $+1 \%$ Sodium Tiossulfate $(\mathrm{P}+\mathrm{N})$ and $0.85 \%$ Saline Solution $+0.5 \%$ Tween 80 $+0.07 \%$ Lecithin $+1 \%$ Sodium Tiosulfate $(\mathrm{SaS}+\mathrm{N})$. The diluent solutions containing exposed and not exposed $S$. Enteritidis SE86 were incubated at $30^{\circ} \mathrm{C}$ and $7{ }^{\circ} \mathrm{C}$ for 1,2 , $3,4,5$ and 6 hours. These incubation times were chosen in order to evaluate regular transport times that generelly are lesser than 6 hours. These temperatures were chosen assuming that they represent temperatures easily performed under controlled $\left(7^{\circ} \mathrm{C}\right)$ and uncontrolled $\left(30^{\circ} \mathrm{C}\right)$ conditions, during the transport of swab samples. The number of colony forming units (cfu) of exposed $S$. Enteritidis SE86 was quantified by the drop technique (Silva et al., 1997). To quantify not exposed Salmonella, $0.1 \mathrm{~mL}$ of each dilution was cultured on the surface of BHI plates. Before incubation for $18-24$ hours at $37^{\circ} \mathrm{C}$, the plates were left to dry at room temperature, inside a laminar flow hood (LabConco, Kansas city, Missouri), previously sterilized by $15 \mathrm{~min}$ of UV exposure. The plates were quantified and the counts were expressed in UFC.mL ${ }^{-1}$. Each measurement was performed in duplicate and each experiment was repeated at least twice.

\section{Evaluation of culture media and plating methods}

Exposed and not exposed S. Enteritidis SE86 cultures were diluted in SaS until $10^{8} \mathrm{cfu} . \mathrm{mL}^{-1}$ and plated $(0.1 \mathrm{~mL})$ in duplicate by surface plating method on TSA (nonselective medium), on four selective media [Mannitol Lysine 
Crystal Violet Brilliant Green Agar (MLCB; Oxoid); Brilliant Green Agar (BGA; Oxoid), Salmonella Shigella Agar (SS; Oxoid) and Xylose Lysine Dextrose (XLD; Oxoid) and on selective media (MLCB, BGA, SS and XLD) overlayed with TSA (TAL method). The cell concentration was determinated by a growth curve. The TAL plates were prepared overlaying $14 \mathrm{~mL}$ of nonselective medium (TSA) onto $25 \mathrm{~mL}$ of each selective medium. The plates were incubated at $37{ }^{\circ} \mathrm{C}$ for $18-24$ hours and bacterial colonies were quantified and expressed in $\mathrm{cfu} \mathrm{mL}^{-1}$.

\section{Evaluation of growth and recovery of $S$. Enteritidis} SE86, S. Typhimurium and S. Bredeney on XLD by different plating methods and culture media

After the choose of the best diluent and the best plating method for the growth of exposed and not exposed $S$. Enteritidis SE86, other two serovars of Salmonella ( $S$. Typhimurium and $S$. Bredeney) were tested by the same techniques, aiming to compare their growth. All the bacteria were individually exposed to $\mathrm{NaDCC}$ and diluted as described previously. After, $0.1 \mathrm{~mL}$ of the dilutions was plated in duplicate on the surface of TSA, XLD and XLD overlayed with TSA (TAL). The plates were incubated for 24 hours at $37^{\circ} \mathrm{C}$. After incubation, the UFC. $\mathrm{mL}^{-1}$ were quantified and the results of each serovar in each medium was evaluated.

\section{Statistical analysis}

All experiments performed to evaluate the growth of microorganisms were carried out at least twice, and all results from counts were made in duplicates. The mean values were calculated and the analysis of variance (ANOVA) and a Tukey Test were carried out to compare the differences between the mean values. The differences were considered significant with $\mathrm{p}$ values were less than 0.05 .

\section{Results}

\section{Multiplication and recovery of $S$. Enteritidis SE86 in different diluents}

The differences between counts of not exposed and exposed $S$. Enteritidis SE86 were approximately $2 \log$ cfu. $\mathrm{mL}^{-1}$, demonstrating the effect of $\mathrm{NaDCC}$ on the bacterial populations.

$S$. Enteritidis SE86 not exposed to NaDCC (Control) showed no significant multiplication $(p<0.05)$ in any of the diluents tested in the first three hours of experiment. However, the diluents $\mathrm{P}+\mathrm{SaS}, \mathrm{P}, \mathrm{SaS}+\mathrm{N}$ and $\mathrm{P}+\mathrm{N}$ sustained significant multiplications (more than $1.5 \mathrm{log}$ ) after four, five, five and six hours at $30^{\circ} \mathrm{C}$, respectively. In opposite, $S$. Enteritidis SE86 did not multiply in the diluent SaS, at $30^{\circ} \mathrm{C}$, during the six hours of incubation, but the cells remained viable. $\mathrm{P}+\mathrm{SaS}$ was the diluent that sustained higher counts of $S$. Enteritidis SE86, increasing $2.0 \mathrm{log}$ UFC. $\mathrm{mL}^{-1}$ after six hours of incubation at $30^{\circ} \mathrm{C}$ (Figure 1).

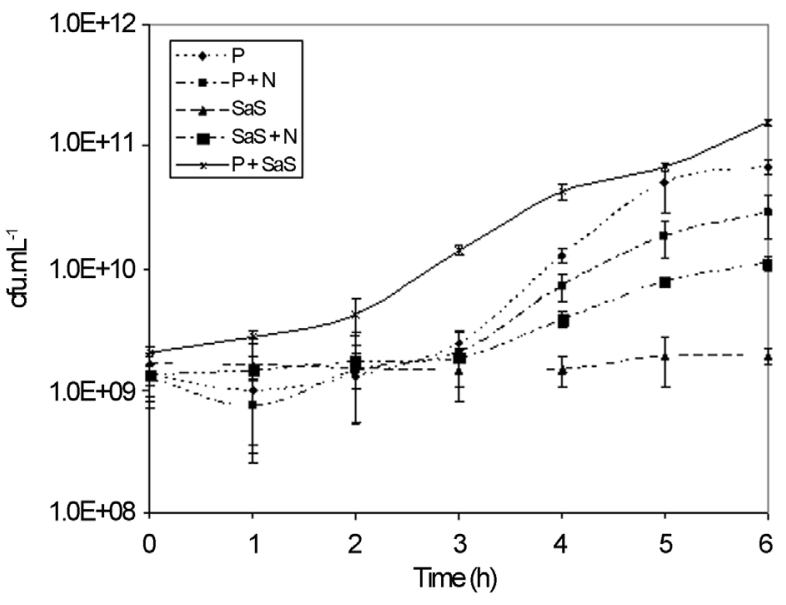

Figure 1 - Multiplication of Salmonella Enteritidis SE86 not exposed to $\mathrm{NaDCC}$ at $30{ }^{\circ} \mathrm{C}$ in Peptone water $(\bullet)$, Saline solution $(\boldsymbol{\Delta})$, Peptone water+Saline solution $(*)$, Peptone water+Tween $80+$ Lecithin+Sodium thiosulfate (*) and Saline solution+Tween 80+Lecithin+Sodium thiosulfate (ם)

$S$. Enteritidis SE86 exposed to NaDCC showed to be stressed because no significant multiplication $(p<0.05)$ in any of the diluents were observed during the six hours at $30{ }^{\circ} \mathrm{C}$, but cells still viable. $S$. Enteritidis SE86 exposed and not exposed to NaDCC showed no significant growth in the diluents $(\mathrm{p}<0.05)$, during the six hours of incubation at $7{ }^{\circ} \mathrm{C}$, however cells remained viable.

\section{Evaluation of culture media and plating methods}

$S$. Enteritidis SE86 not exposed and exposed to NaDCC showed no significant differences $(\mathrm{p}<0.05)$ among counts on non selective media (TSA), on selective media (BGA, SS XLD and MLCB) and on selective media XLD, SS, BGA overlayed with TSA (TAL media). TSA counts were higher than counts observed on selective media and TAL media, but this difference was not significant. MLCB overlayed with TSA demonstrated lower counts when compared with other TAL media.

Recovery of $S$. Enteritidis SE86, S. Typhimurium and $S$. Bredeney grown in XLD by different plating methods

The three serovars $S$. Enteritidis SE86, $S$. Typhimurium and $S$. Bredeney, not exposed (Figure 2) and exposed (Figure 3 ) to NaDCC showed no significant difference in counts on non selective TSA, on selective medium XLD and XLD overlayed with TSA (TAL).

\section{Discussion}

In the present study, $S$. Enteritidis SE86 was able to grow at $30^{\circ} \mathrm{C}$ in all diluents tested, except $\mathrm{SaS}$. In order to quantify microorganisms present in a diluted sample, an appropriate diluent should not promote bacterial multiplication or death, should be ease to prepare, should present low 


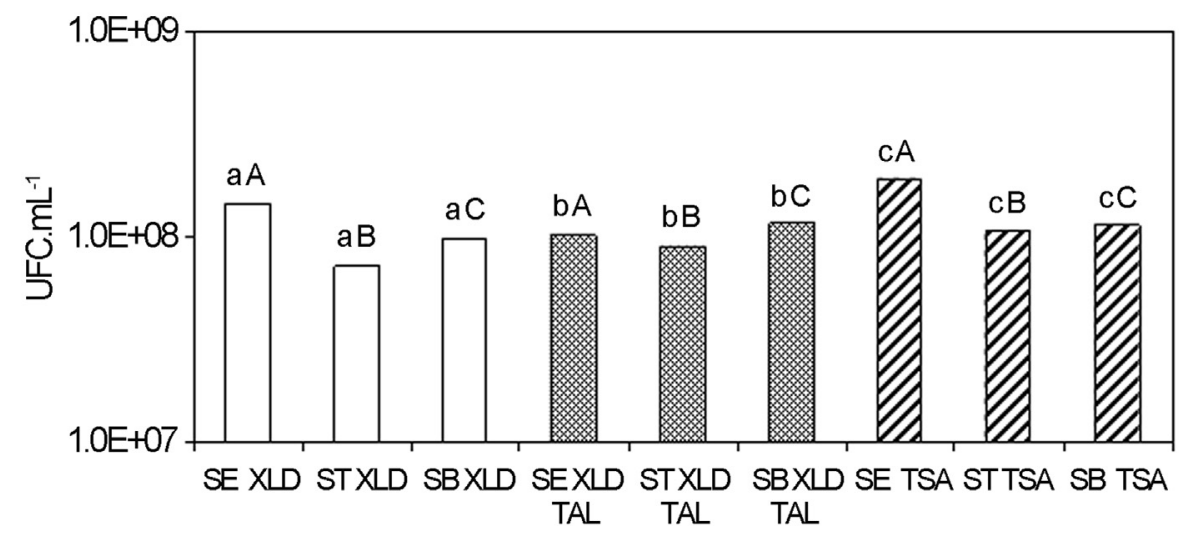

Figure 2 - Multiplication of Salmonella Enteritidis SE86 (SE), Salmonella Typhimurium (ST) and Salmonella Bredeney (SB) not exposed to sodium dichloroisocyanurate plated on XLD, XLD overlayed with TSA (TAL) and TSA. Values with different letters differ significantly (p $<0,05)$. a,b,c: Comparison among serovars on the same medium plated by the same method. A, B, C: Comparison of the growth of the same Salmonella serovar inoculated on different media.

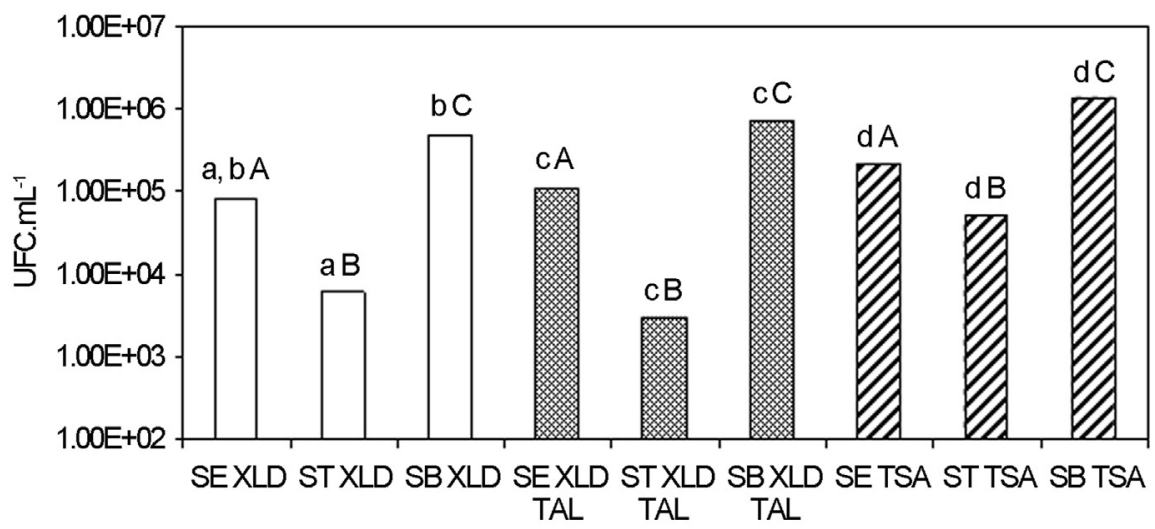

Figure 3 - Multiplication of Salmonella Enteritidis SE86 (SE), Salmonella Typhimurium (ST) and Salmonella Bredeney (SB) exposed to sodium dichloroisocyanurate for $5 \mathrm{~min}$ and plated on XLD, XLD overlayed with TSA (TAL) and TSA. Values with different letters differ significantly ( $\mathrm{p}<0,05$ ). a,b,c: Comparison among serovars on the same medium plated by the same method. A, B, C: Comparison of the growth of the same Salmonella serovar inoculated on different media.

cost, and ideally, should be able to recover stressed cells (Wu et al., 2001; Wu, 2008). Based on these appointments and according to our results, The most appropriate diluent was $\mathrm{SaS}$, because it did not cause decreasing in the $\mathrm{NaDCC}$ exposed cell numbers and did not sustain the multiplication of not exposed cells. Additionally, this diluent is easy to be prepared and have a lower cost when compared to other diluents.

After the exposure to NaDCC, S. Enteritidis SE86 showed no significant multiplication in any of the diluents tested, even after six hours of incubation at $30^{\circ} \mathrm{C}$. As no significant multiplication occurred in any of the diluents, the cell recovery was not assessed, and it was not possible to distinguish dead cells from stressed or viable but not cultivable (VNC) cells. In order to recover stressed cells, bacteria present an extension in its lag phase, and this is called the recovery period. After this period, cells start to multiply at an equal rate to the not stressed cells (Tomlins and Ordal,
1971). As an example, a study have demonstrated a four to five hours lag phase of $S$. Typhimurium growing in Trypticase Soy Broth (TSB) and on TSA, after heat stress at $48^{\circ} \mathrm{C}$ for 30 min (Clark et al., 1968; Clark and Ordal, 1969). The experiments conducted in this study may suggest that the extent of the lag phase of $S$. Enteritidis SE86 exposed to NaDCC became longer than six hours, because not exposed present SE86 presented shorter lag phases in different diluents. It is possible that longer periods of incubation could be able to show the exact duration of the lag phase necessary to recover the stressed cells. However, it was not the purpose of this work, since the time of six hours was chosen because it is often recommended as an appropriate period of sample transport inside diluents (Silva et al., 1997; Lightfoot and Maier, 2003).

At $7{ }^{\circ} \mathrm{C}$, not exposed $S$. Enteritidis SE86 (Control) showed no significant growth in the diluents, during the six hours of incubation. Similar results demonstrated that pure 
Buffered Peptone Water and also added with Tween $80+$ Lecithin and Tween 80 + Lecithin did not sustain multiplication of Bacillus cereus and Escherichia coli, at $4{ }^{\circ} \mathrm{C}$, during 6 hours (Greig and Ravel, 2009). In other study, no significant increase in the numbers of cells of Salmonella spp., Escherichia coli O157:H7 and Staphylococcus aureus was observed during nine hours of storage at $4{ }^{\circ} \mathrm{C}$, after disinfection of shredded cabbage, using $0.1 \%$ calcined calcium, for $20 \mathrm{~min}$, combined with $100 \mathrm{mg} \mathrm{kg}^{-1}$ of Sodium hypochlorite, for 20 min (Fukuyama et al., 2009). The results of the present study suggest that the temperature of $7^{\circ} \mathrm{C}$ can be used to transport samples without allowing the multiplication of $S$. Enteritidis SE86. It is possible that this result may be valid for other microorganisms, since the American Public Health Association recommended refrigeration temperature for the transport of bacterial samples collected by swabs from surfaces, but the exact temperature is not define (APHA, 2001).

When the culture media and the plating methods were evaluated, no significant difference was shown in the counts of $S$. Enteritidis SE86 exposed to NaDCC, however there were increased numbers on TSA when compared with selective media and TAL. This quantitative difference suggests that there was few stressed cells, but the selective media did not inhibit significantly the multiplication of $S$. Enteritidis SE86, making it suitable for its cultivation, even by direct plating. Corroborating this result, the ISO $6579 / 2002$ recommends the use of XLD plates for the enumeration of Salmonella.

In the present study, selective media overlayed with TSA (TAL) also showed no significant difference in counts of $S$. Enteritidis SE86 exposed to NaDCC, when compared with TSA or selective media. This result is interesting, since the TAL method has been reported as important for the recovery of stressed bacterial cells (Osaili et al., 2010; Kang and Fung, 1999; Kang and Fung, 2000). According to the present work, XLD showed no significant difference in counts obtained on non-selective TSA and selective media overlayed with TSA, with or without NaDCC exposure. XLD plates are recommended by the Brazilian Ministry of Agriculture (MAPA) for Salmonella investigation and by ISO 6579/2002 for Salmonella quantification, suggesting its adequacy to be used in the enumeration of stressed $S$. Enteritidis SE86, at least for in vitro experiments. However, in order to evaluate its adequacy to real surface or food samples, more experiments are necessary.

When the growth and recovery of $S$. Enteritidis SE86, $S$. Typhimurium and $S$. Bredeney were evaluated, no significant differences in counts on the XLD plates, non-selective TSA and TAL were observed, demonstrating that the selective media did not inhibit the growth of these strains of Salmonella. This result is interesting and corroborates that such media could be suitable for the quatification of Salmonella exposed or not exposed to NaDCC. Other studies are necessary to evaluate the behavior of these microorganisms, stressed by disinfectants on surfaces of food services.

In conclusion, this study indicated that $0.85 \%$ Saline $(\mathrm{SaS})$ solution did not support $S$. Enteritidis SE86 growth and maintain cells viable for six hours, suggesting being an adequate diluent for the collection and transport of swab samples for the investigation of $S$. Enteritidis SE86. The temperature of $7{ }^{\circ} \mathrm{C}$ demonstrated to be able to maintain stable the numbers of $S$. Enteritidis SE86, suggesting to be adequate for the transport of swab samples. Finally, the direct plating on XLD medium demonstrated the same Salmonella counts that non selective TSA and TAL media, suggesting to be adequate for the quantitative analysis of different Salmonella serovars, exposed or not exposed to $\mathrm{NaDCC}$, at least in laboratory conditions. Further studies are necessary to test these procedures for the analysis of Salmonella in natural conditions.

\section{Acknowledgments}

The authors would like to thank Dr. Marisa Ribeiro de Itapema Cardoso for kindly providing the strains $S$. Typhimurium and $S$. Bredeney. We are greatfull to the Ministry of Education for providing the scholarship that made the present work possible. We also thank to Kalykim for providing the disinfectant that was used in the experiments. The authors would like to thank to Prof. Dra. Florencia Cladera from ICTA/UFRGS for the statistical analyses.

\section{References}

APHA - American Public Health Association (2001) Compendium of Methods for the Microbiological Examinations of Foods, $4^{\text {th }}$ edition. Frances Pouch Downes and Keith Ito Washington, D.C.

Brasil (2011) Análise epidemiológica dos surtos de doenças transmitidas por alimentos no Brasil, 1999-2011. Available: http://portal.saude.gov.br/portal/arquivos/pdf/analise_ep_ surtos_dta_brasil_2011.pdf. Accessed November 4, 2011.

Clark CW, Witter LD, Ordal ZJ (1968) Thermal Injury and Recovery of Streptococcus faecalis. Appl Microbiol 16:17641769.

Clark CW, Ordal ZJ (1969) Thermal injury and recovery of Salmonella Typhimurium and its effect on enumeration procedures. Appl Microbiol 18:332-333

Cunha B (2008) Investigação de Surtos Alimentares ocorridos em Serviços de Alimentação no Rio Grande Do Sul. (Trabalho de conclusão de curso de Graduação - Engenharia de Alimentos, Instituto de Ciência e Tecnologia de Alimentos, Universidade Federal do Rio Grande do Sul, Porto Alegre, RS, Brasil).

Fukuyama S, Watanabe Y, Kondo N, Nishinomyia T, Kawamoto S, Isshiki K, Murata M (2009) Efficiency of Sodium Hipoclorite and Calcinated Calcium in killing Escherichia coli O157:H7, Salmonella spp. and Staphylococcus aureus attached to freshly shredded cabbage. Biosci Biotechnol Biochem 73:9-14.

Geimba MP, Tondo EC, Oliveira FA, Canal CW, Brandelli A (2004) Serological characterization and prevalence of spvR 
genes in Salmonella sp. isolated from foods involved in foodborne outbreaks occurred in Rio Grande do Sul, South of Brasil. J Food Prot 67: 1229-1233.

Greig JD, Ravel A (2009) Analysis of foodborne outbreak data reported internationally for source attribution. Int $\mathrm{J}$ Food Microbiol 130: 77-78.

Jasson V, Debevere J, Rajkovic A, Uyttendaele M (2007) Establishment of procedures provoking sub-lethal injury of Listeria monocytogenes, Campylobacter jejuni and Escherichia coli $\mathrm{O} 157: \mathrm{H} 7$ to serve method performance testing. Int J Food Microbiol 118: 241-249.

Kang DH, Fung DYC (2000) Thin Agar Layer Method for Recovery of Salmonella Typhimurium. Int J Food Microbiol 54:127-132.

Kang DH, Fung DYC (1999) Thin Agar Layer Method for Recovery of Heat-Injured Listeria monocytogenes. J Food Prot 62:1346-1349.

Lightfoot NF, Maier EA (2003) Análise microbiológica de alimentos e água. Lisboa: Fundação Calouste Gulbenkian $284 \mathrm{pp}$.

Malheiros PS, Brandelli A, Noreña CPZ, Tondo EC (2009) Acid and thermal resistance of a Salmonella Enteritidis strain involved in several foodborne outbreaks. J Food Saf, 29:302317.

Moore G, Griffith C (2007) Problems associated with traditional hygiene swabbing: the need for in-house standardization. J Appl Microbiol, 103:1090-1103.

Oliveira FA, Pasqualoto AP, Silva WP, Tondo EC (2012) Characterization of Salmonella Enteritidis isolated from human samples. Food Res Int 45:1000-1003.

Osaili TM, Al-Nabulsi AA, Shaker RR, Al-Holy MM, Al-Haddaq MS, Olaimat NA, Ayyash MM, Al Ta'ani MK, Forsythe S J (2010) Efficacy of the Thin Agar Layer Method for the Recovery of Stressed Cronobacter spp. (Enterobacter sakazakii). J Food Prot 73:1913-1918.
Peng J, Tsai W, Chou C (2002) Inactivation and removal of Bacillus cereus by sanitizers and detergent. Int J Food Microbiol 77:11-18.

Rossoni EM, Gaylarde CC (2000) Comparison of sodium hypochlorite and peracetic acid as sanitising agents for stainless steel food processing surfaces using epifluorescence microscopy. Int J Food Microbiol 61:81-85.

Silva N, Junqueira VCA, Silveira NFA (1997) Manual de Métodos de Análise Microbiológica de Alimentos. $1^{\text {th }}$ edition. Editora Varela, São Paulo, 295 pp.

Tomlins R, Ordal ZJ (1971) Precursor ribosomal ribonucleic acid and ribosome accumulation in vivo during the recovery of Salmonella Typhimurium from thermal injury. J Bacteriol 107:134-142.

Tondo EC, Machado TRM, Malheiros PS, Padrão DK, Carvalho AL, Brandelli A (2010) Adhesion and biocides inactivation of Salmonella on stainless steel and polyeyhilene. Braz J Microbiol 41:1027-1037.

Wu VCH, Fung DYC, Kang DH, Thompson LK (2001) Evaluation of Thin Agar Layer Method for Recovery of AcidInjured Foodborne Pathogens. J Food Prot 64:1067-1071.

Wu VCH, Fung DYC (2006) Simultaneous recovery and detection of four heat-injured foodborne pathogens in ground beef and milk by a four-compartment thin agar layer plate. J Food Saf 26:126-136.

Wu VCH (2008) A review of microbial injury and recovery methods in food. Food Microbiol 25:735-744.

Tondo EC, Ritter AC, (2012) Salmonella and Salmonellosis in Southern Brazil: a review of the last decade. In: Salmonella: Classification, Genetics and Disease outbreaks, Monte A.S., De Santos P.E. (eds). Nova Science Publishers, Inc. New York, pp 175-191.

All the content of the journal, except where otherwise noted, is licensed under a Creative Commons License CC BY-NC. 